\title{
ESTABILIDADE DO ÁCIDO ASCÓRBICO EM IOGURTE DE LEITE DE BÚFALA ADICIONADO DE DIFERENTES CONCENTRAÇÕES DE POLPA DE CAMU-CAMU (Myrciaria dubia)
}

\author{
Stability of ascorbic acid in buffalo milk yogurt with camu-camu \\ (Myrciaria dubia) in different concentrations
}

\begin{abstract}
Raiane Conceição Sarmento ${ }^{I}$, Maria Raiane Machado Pinto ${ }^{I}$, Rayana Silva Craveiro ${ }^{I}$, Endril Pablo Brasil de Freitas ${ }^{1}$, Elivaldo Nunes Modesto Junior ${ }^{2 *}$, Rafael Vitti Mota ${ }^{1}$
\end{abstract}

\section{RESUMO}

O camu-camu (Myrciaria dubia) é um fruto da região amazônica, natural das margens alagadas, possui formato globoso, de superfície lisa e brilhante, de cor vermelha escura até preto púrpura durante o estágio de maturação. Possui teor de ácido ascórbico que varia de 800 a $6.100 \mathrm{mg} / 100 \mathrm{~g}$ na polpa fresca, no entanto, por apresentar uma elevada acidez, o seu consumo se torna inviável, tendo como alternativa de consumo a sua incorporação nos derivados lácteos, como iogurte. Dessa forma, o objetivo deste estudo foi avaliar a estabilidade do ácido ascórbico durante o período de armazenamento de iogurtes de leite bubalino com diferentes concentrações de polpa de camu-camu. Foram avaliadas as características físico-químicas da polpa e do iogurte de leite de búfala elaborado a partir de duas formulações com $8,3 \%$ (F1) e $12,5 \%$ (F2) de polpa de camu-camu, além da estabilidade do ácido ascórbico no produto armazenado sob refrigeração $\left(5 \pm 1{ }^{\circ} \mathrm{C}\right)$ por 28 dias. A polpa analisada e as formulações de iogurte apresentaram características físico-químicas conforme previsto na literatura e preconizado pela legislação vigente. O teor de ácido ascórbico nas formulações foi de 242,2 mg/100 g (F1) e 317,73 mg/100 g (F2) no tempo zero e de $171,0 \mathrm{mg} / 100 \mathrm{~g}$ (F1) e 242,2 mg/100 g (F2), no $28^{\circ}$ dia de armazenamento, havendo uma perda de $30 \%$ no teor de ácido ascórbico durante todo o período de armazenamento.

Palavras-chave: iogurte bubalino; vitamina $\mathrm{C}$; antocianina.

1 Universidade do Estado do Pará, Centro de Ciências Naturais e Tecnologia, Belém, PA, Brasil.

2 Universidade Federal do Pará, Campus Universitário do Guamá, Instituto de Tecnologia, Rua Augusto Corrêa, 01, Centro, 66.075-110, Belém, PA, Brasil. E-mail: modesto.ufpa@gmail.com

* Autor para correspondência 


\begin{abstract}
The camu-camu (Myrciaria dubia) is a fruit of the Amazonian region, natural of the flooded margins, has a globose shape, smooth and bright surface, dark red to purple-black colour during the maturation stage. It has ascorbic acid content ranging from 800 to $6,100 \mathrm{mg} / 100 \mathrm{~g}$ in the fresh pulp, however, because it presents a high acidity, its consumption in natura becomes unfeasible, having as alternative consumption its incorporation in dairy derivatives such as yogurt. The objective of this study was to evaluate the stability of ascorbic acid in buffalo milk yogurt with different concentrations of camu-camu pulp during the storage period. It was evaluated the physicochemical characteristics of the pulp and yogurt of buffalo milk prepared from two formulations with $8.3 \%$ (F1) and $12.5 \%$ (F2) of camu-camu pulp, besides the stability of ascorbic acid in the product stored under refrigeration $\left(5 \pm 1{ }^{\circ} \mathrm{C}\right.$ ) for 28 days. The pulp analyzed and the yogurt formulations presented physicochemical characteristics as predicted in the literature and recommended by the current legislation. The ascorbic acid contents in the formulations were 242.2 $\mathrm{mg} / 100 \mathrm{~g} \mathrm{(F1)} \mathrm{and} 317.73 \mathrm{mg} / 100 \mathrm{~g} \mathrm{(F2)} \mathrm{at} \mathrm{zero} \mathrm{time} \mathrm{and} 171.0 \mathrm{mg} / 100 \mathrm{~g}$ (F1) and $242.2 \mathrm{mg} / 100 \mathrm{~g}$ (F2) on the $28^{\text {th }}$ day of storage, with a $30 \%$ loss in ascorbic acid content during the entire storage period.
\end{abstract}

Keywords: buffalo yogurt; vitamin C; anthocyanin.

\section{INTRODUÇÃO}

Na Amazônia existem inúmeras espécies vegetais com potencial econômico, dentre as quais se destaca o camu-camu (Myrciaria dubia (H.B.K.) McVaugh), uma Myrtaceae, silvestre, encontrada nas margens de rios e lagos da Amazônia (RIBEIRO et al., 2002). Este fruto destaca-se por apresentar um considerável teor em ácido ascórbico, em relação a outras frutas, cerca de 6.000 $\mathrm{mg} / 100 \mathrm{~g}$ na polpa fresca, o que representa aproximadamente duas vezes mais ácido ascórbico que na acerola e 60 vezes mais que na laranja, além de conter elevada concentração de antocianinas e minerais como potássio, cálcio e zinco (PINEDO, 2007; RODRIGUES; MARX, 2006; YUYAMA et al., 2002).

O consumo de camu-camu, Myrciaria dubia, é crescente em diferentes países e internacionalmente a fruta posicionouse na preferência dos consumidores de frutas tropicais exóticas, especialmente por apresentar em sua composição o ácido ascórbico (FUJITA et al., 2017). A presença deste composto despertou o interesse de importadores do Japão, Europa e EUA, sendo o Peru o principal exportador (PINEDO, 2007). Este fruto é utilizado principalmente na forma de suco e néctar. Porém, sabe-se que a concentração e estabilidade desta vitamina varia com a espécie, estado de maturação, tempo e temperatura de processamento, $\mathrm{pH}$ e presença de oxigênio e enzimas. Apesar dessa vantagem, o sabor amargo e a elevada acidez da polpa do camu-camu limitam o seu consumo, dessa forma, o seu processamento torna-se uma alternativa para minimizar o sabor acentuado. Uma das formas indicadas para aproveitar os benefícios advindos da polpa de camu-camu é adicionar este a derivados lácteos como o iogurte (MAEDA et al., 2007).

O leite de búfala, comparado ao leite de vaca, apresenta maior teor de proteína e contém micelas de caseína mais volumosas, o que lhe confere rápida coagulação no 
processamento de derivados com menos soro, gerando produtos de maior firmeza. Seu elevado teor de lipídios promove um maior rendimento na produção de seus derivados, constituindo-se de glóbulos maiores de coloração clara. Os ácidos capróico, caprílico e cáprico, apesar de serem encontrados em menor quantidade, quando liberados nos produtos lácteos conferem sabor e aroma característicos (MESQUITA et al., 2001).

O iogurte de leite de búfala é um produto alimentício com um considerável valor nutricional, proveniente de processo fermentativo por bactérias específicas, que apresenta em sua composição teores de ácido fólico, vitamina A, vitaminas do complexo B e sais minerais. É tido como alimento funcional, visto que seu consumo traz consigo diversos benefícios para a saúde (PIMENTEL et al., 2005). Oferece fácil digestão por indivíduos com distúrbios intestinais, desde que tal produto seja de qualidade, apresentando baixa contagem de microrganismos, características desejáveis de sabor e textura, e segurança alimentar aos consumidores (OLIVEIRA et al., 2008).

Dessa forma, verifica-se a necessidade de estudos científicos que certifiquem se os produtos lácteos elaborados a partir do leite de búfala e do acréscimo de frutas possuem propriedades nutricionais e funcionais, além de características e sabores especiais que possam não somente atrair os consumidores, mas também trazer relevantes contribuições para sua saúde. Diante disso, o presente estudo objetivou avaliar a estabilidade do ácido ascórbico durante o período de armazenamento de iogurte de leite bubalino com diferentes concentrações de polpa de camu-camu.

\section{MATERIAL E MÉTODOS}

Os frutos utilizados na elaboração do iogurte de leite de búfala foram provenientes de uma propriedade rural, localizada no município de São João de Pirabas, na região nordeste do estado do Pará, Brasil. A colheita foi realizada manualmente, os frutos foram selecionados visualmente quanto ao grau de maturação, aparência e sanidade. Utilizaramse somente os frutos sadios e meio-maduros, ou seja, de coloração vermelho-claro púrpura. Os mesmos foram transportados ao local de processamento (Laboratório de Tecnologia de Alimentos da Universidade Estadual do Pará - UEPA, Campus Salvaterra, Pará, Brasil) em caixas térmicas com bolsas de gelo, para minimizar possíveis alterações em sua qualidade.

Para o processamento, os frutos foram sanitizados com solução de hipoclorito de sódio a $150 \mathrm{ppm}$, por $15 \mathrm{~min}$, posteriormente lavados com água corrente e despolpados em despolpadeira de aço com malha de $1,5 \mathrm{~mm}$ de abertura. A polpa foi pasteurizada e acondicionada a frio em embalagens de polietileno com capacidade para $500 \mathrm{~mL}$ e armazenada sob congelamento a $-18{ }^{\circ} \mathrm{C}$, até o momento de sua utilização.

A polpa do camu-camu foi avaliada em triplicata quanto às características de umidade, cinzas, sólidos solúveis, $\mathrm{pH}$, acidez total titulável pelos métodos descritos por AOAC (HORWITZ; LATIMER, 2006), teor de ácido ascórbico (NOJAVAN et al., 2008) e teor de antocianinas (LEE; FRANCIS, 1972). Para o desenvolvimento do iogurte, utilizouse o leite de búfala obtido em propriedades rurais do município de Soure, Pará, Brasil. O leite de búfala utilizado no experimento foi obtido manualmente de fêmeas bubalinas da raça Murrah, em condições higiênicas adequadas.

Após a ordenha, o leite foi imediatamente resfriado a $5{ }^{\circ} \mathrm{C}$ em tanque de expansão, sendo coletado em garrafas de polipropileno de 1 litro e transportado em caixa térmica até o laboratório, onde foram realizados os processamentos. Foi utilizada cultura láctea 
termófila composta de cepas de Streptococcus thermophilus e Lactobacillus delbrueckii subsp. bulgaricus. A cultura "mãe" foi preparada pelo método de semeadura do cultivo liofilizado em um litro de leite de búfala previamente aquecido $\left(90{ }^{\circ} \mathrm{C}\right.$ por 30 minutos), seguido de incubação $\left(42{ }^{\circ} \mathrm{C}\right.$ por três horas), até alcançar o valor de $\mathrm{pH}$ de 4,5. Esta cultura era mantida a $5{ }^{\circ} \mathrm{C}$, sendo utilizada para o preparo do cultivo no dia de produção do iogurte, através da semeadura de $2 \%$ em um litro de leite de búfala. Utilizou-se para o desenvolvimento do iogurte a polpa de camu-camu previamente preparada e açúcar refinado adquirido no comercio local.

Para o processamento do iogurte, transferiu-se o leite de búfala para um recipiente de aço inox, sendo o mesmo aquecido a $90{ }^{\circ} \mathrm{C}$ por 15 minutos e, em seguida, resfriado até $43{ }^{\circ} \mathrm{C}$. Adicionou-se o cultivo na proporção de $2 \%(\mathrm{v} / \mathrm{v})$ do leite de búfala, posteriormente homogeneizou-se e submeteu-se ao processo de fermentação do produto à temperatura de $43{ }^{\circ} \mathrm{C}$ por aproximadamente 6 horas. Após este período, o produto foi submetido novamente ao processo de resfriamento, desta vez à uma temperatura de $10{ }^{\circ} \mathrm{C}$, adicionando-se então a e polpa de camu-camu e o açúcar. Foram processadas duas formulações de iogurte de leite de búfala com polpa de camu-camu, diferindo-se na concentração de polpa, sendo $8,3 \%$ (formulação F1) e 12,5\% (formulação F2), mantendo a concentração de açúcar (12\%), para as duas formulações. Os produtos foram agitados manualmente com haste de aço inox durante 5 minutos e embalado em garrafas de polietileno com capacidade de $500 \mathrm{~mL}$.

As formulações de iogurte foram avaliadas quanto aos parâmetros de $\mathrm{pH}$, acidez total titulável, umidade, cinzas e sólidos solúveis, conforme descritos por AOAC (HORWITZ; LATIMER, 2006). Foi avaliada a estabilidade do ácido ascórbico no iogurte de leite de búfala acrescido da polpa do camucamu, mantido sob refrigeração $\left(5 \pm 1{ }^{\circ} \mathrm{C}\right)$ por 28 dias, sendo coletadas amostras a cada 4 dias de armazenamento. O teor de ácido ascórbico foi quantificado conforme descrito por Nojavan et al., 2008.

Os dados obtidos foram analisados por meio da análise de variância (ANOVA), comparando-se as médias pelo teste de Tukey com nível de $5 \%$ de significância $(\mathrm{p}<0,05)$.

\section{RESULTADOS E DISCUSSÃO}

Na Tabela 1 estão apresentados os resultados da caracterização físico-química da polpa de camu-camu, quanto aos parâmetros $\mathrm{pH}$, acidez total, umidade, cinzas, sólidos solúveis, antocianinas totais e ácido ascórbico.

Tabela 1 - Valores obtidos para caracterização físico-química da polpa de camu-camu

\begin{tabular}{lc}
\hline \multicolumn{1}{c}{ Parâmetros } & Média \\
\hline $\mathrm{pH}$ & $2,90 \pm 0,01$ \\
Acidez total (\% ácido cítrico) & $2,04 \pm 0,03$ \\
Umidade (\%) & $89,30 \pm 0,22$ \\
Cinzas (\%) & $7,55 \pm 0,20$ \\
Sólidos solúveis ( $\left.{ }^{\circ} \mathrm{Brix}\right)$ & $4,33 \pm 0,29$ \\
Antocianinas totais $(\mathrm{mg} / 100 \mathrm{~g})$ & $6,09 \pm 0,01$ \\
Ácido ascórbico $(\mathrm{mg} / 100 \mathrm{~g})$ & $1.839,93 \pm 6,70$ \\
\hline
\end{tabular}

A avaliação da polpa quanto ao $\mathrm{pH}$, acidez total e umidade demonstram que os valores obtidos estão de acordo com aqueles citados na literatura (BARRETO et al., 2013; SOUZA et al., 2013; OLIVEIRA et al., 2013; OLIVEIRA et al., 2014; FUJITA et al., 2017). Já o teor de sólidos solúveis foi inferior quando comparado com os valores obtidos pelos mesmos autores citados acima. O teor de cinzas foi pouco identificado nas literaturas, sendo o valor obtido no presente estudo superior $(7,55 \pm 0,20 \%)$ ao descrito por 
Marinho et al. (2009) $(3,00 \mathrm{~g} / 100 \mathrm{~g})$. O teor de antocianinas $(6,09 \pm 0,01 \mathrm{mg} / 100 \mathrm{~g})$ foi superior ao descrito por Marques (2006) $(5,70$ $\mathrm{mg} / 100 \mathrm{~g})$ e inferior ao citado por Marinho et al. (2009) $(9,96 \mathrm{mg} / 100 \mathrm{~g})$. Segundo Oliveira (2014), a síntese das antocianinas ocorre naturalmente pelo metabolismo da planta com a evolução da maturação dos frutos. A polpa de camu-camu apresentou elevada concentração de ácido ascórbico $(1.839,93 \pm 6,70 \mathrm{mg} / 100 \mathrm{~g})$, sendo superior ao descrito por Barreto et al. (2013) $(1.236,12 \mathrm{mg} / 100 \mathrm{~g})$ e inferior ao descrito por Maeda et al. (2007) $(2.585,40$ $\mathrm{mg} / 100 \mathrm{~g}$ ).

Na Tabela 2 estão apresentados os resultados encontrados para análise físicoquímica das formulações F1 e F2 de iogurte de leite de búfala com polpa de camu-camu, avaliadas no tempo zero.

A partir dos resultados apresentados observou-se que os parâmetros analisados apresentaram diferença significativa $(\mathrm{p}<0,05)$ entre as formulações, com exceção dos sólidos solúveis. Cunha Neto et al. (2005) relataram em sua pesquisa o valor de 4,41 de $\mathrm{pH}$ para iogurte integral de leite bubalino, valor próximo aos encontrados no presente estudo $(4,35 \pm 0,01$ e 4,25 $\pm 0,01)$. Modesto
Júnior (2016) ao estudar o iogurte grego de leite de búfala com diferentes concentrações $(10,20$ e $30 \%)$ de calda de ginja, encontrou valores de $\mathrm{pH}$ inferiores ao do presente estudo com variação de 3,90 a 3,36 sendo estes resultados relacionados ao tipo de fruta adicionado ao produto. A análise do $\mathrm{pH}$ no produto é de extrema relevância, visto que é um fator determinante em seu tempo de deterioração, por meio do desenvolvimento de microrganismos (LIMA, 2013).

Para acidez total, apesar da diferença estatística $(p<0,05)$, os valores obtidos entre as formulações foram próximos (F1 0,94 \pm $0,01 \%$ e F2 $1,04 \pm 0,01 \%$ ), ressalta-se que estes resultados foram similares aos valores descritos por Borges et al. (2009), que ao analisarem o iogurte de leite de búfala sabor cajá obtiveram $0,98 \%$ e Rocha et al. (2004) que ao avaliar a acidez de iogurte de leite de búfala com mangaba encontraram $1,07 \%$. As formulações apresentaram-se em conformidade com a legislação vigente que permite acidez em iogurte variando de 0,6 a 2,0\% em ácido lático (BRASIL, 2007). De acordo com Santos (2015) a variação de acidez do iogurte pode ocorrer conforme a temperatura de incubação, sendo essencial

Tabela 2 - Resultados obtidos para as análises físico-químicas das formulações F1 com 8,3\% de polpa e F2 com 12,5\% de polpa de camu-camu, avaliadas no tempo zero

\begin{tabular}{|c|c|c|}
\hline \multirow{2}{*}{ Parâmetros } & \multicolumn{2}{|c|}{ Formulações* } \\
\hline & F1 & F2 \\
\hline $\mathrm{pH}$ & $4,35^{\mathrm{a}} \pm 0,01$ & $4,25^{\mathrm{b}} \pm 0,01$ \\
\hline Acidez total (\% ácido lático) & $0,94^{\mathrm{b}} \pm 0,01$ & $1,04^{\mathrm{a}} \pm 0,01$ \\
\hline Umidade (\%) & $73,13^{b} \pm 0,40$ & $73,75^{\mathrm{a}} \pm 0,10$ \\
\hline Cinzas $(\%)$ & $9,25^{\mathrm{b}} \pm 0,12$ & $11,30^{\mathrm{a}} \pm 0,06$ \\
\hline Sólidos solúveis ( ${ }^{\circ}$ Brix) & $22,67^{\mathrm{a}} \pm 1,15$ & $21,33^{\mathrm{a}} \pm 1,15$ \\
\hline Ácido ascórbico (mg/100 g) & $242,2^{b} \pm 3,81$ & $317,73^{\mathrm{a}} \pm 3,92$ \\
\hline
\end{tabular}

* Letras diferentes, na mesma linha, indicam diferenças significativas pelo teste de Tukey, $(\mathrm{p}<0,05)$. 
para que haja o desenvolvimento da cultura durante o armazenamento a frio, além disso, a alteração na acidez também pode estar diretamente relacionada com a fruta que foi acrescida a sua formulação.

Os valores de umidade das formulações F1 $(73,13 \pm 0,40 \%)$ e F2 $(73,75 \pm 0,10 \%)$ são similares aos descritos por Soares et al. (2016) que ao estudarem o iogurte tipo sundae sabor bacuri obtiveram valores de $73,1 \%$ a $78,0 \%$. Já Paiva et al. (2015) encontraram valores de umidade superiores ao do presente estudo (76,4 a 79,6\%) nas diferentes formulações de iogurte adicionado de polpa de abacaxi com mel. Quanto aos teores de cinzas no iogurte, os valores encontrados neste estudo F1 $(9,25$ $\pm 0,12 \%)$ e F2 $(11,30 \pm 0,06 \%)$, foram bem superiores aos descritos na literatura, como os citados por Paiva et al. (2015) (0,99\%) em iogurte adicionado de polpa de abacaxi com mel, por Costa et al. (2012) (0,66\%) em iogurte saborisado com $7 \%$ de polpa de juçaí (Euterpe edulis Martius) e por Oliveira et al. (2011) (1,25\%) em iogurte de açaí tipo sundae. Conforme Santos (2015) a umidade de um alimento tem relação com sua estabilidade, qualidade e composição e pode alterar fatores como armazenamento e o processamento.

Os teores de sólidos solúveis no iogurte para as formulações do presente estudo foram $22,67 \pm 1,15{ }^{\circ}$ Brix para F1 e $21,33 \pm 1,15^{\circ}$ Brix para F2, estes resultados mostraram-se superiores aos valores descritos por Costa et al. (2012) que em sua pesquisa relata uma variação de 13,44 a $14,11^{\circ}$ Brix. Já Paiva et al. (2015) relata valores inferiores de 37,1 a $41,5^{\circ}$ Brix. Maeda et al. (2006), ao estudarem o néctar de camu-camu, obtiveram $19,93{ }^{\circ}$ Brix, sendo um indicativo de que os sólidos solúveis sejam intrínsecos ao tipo de polpa utilizada para saborizar o iogurte.

Os teores de ácido ascórbico das formulações (F1 242,2 \pm 3,81 mg/100 g e F2
$317,73 \pm 3,92 \mathrm{mg} / 100 \mathrm{~g}$ ) foram superiores aos valores reportados por Rosa (2011) ao elaborar iogurte de leite de búfala adicionado com polpas de frutas da Amazônia, tais como camu-camu $(202,31 \mathrm{mg} / 100 \mathrm{~g})$ e com mix de frutas como acerola com camu-camu $(210,04 \mathrm{mg} / 100 \mathrm{~g})$, bacuri com camucamu $(140,13 \mathrm{mg} / 100 \mathrm{~g})$ e cupuaçu com camu-camu $(140,22 \mathrm{mg} / 100 \mathrm{~g})$, indicando que as formulações de iogurte do presente estudo concentram boa quantidade do ácido ascórbico advindo da polpa do camu-camu.

$\mathrm{Na}$ Tabela 3 estão apresentados os resultados das alterações no teor de ácido ascórbico para as formulações F1 e F2 durante o período de armazenamento sob refrigeração por 28 dias, analisados em intervalos de 4 dias. Comparando-se as formulações entre si, observou-se para F1 que as amostras que não apresentaram diferença significativa foram somente as dos tempos (0 e 4 dias) e ( 24 e 28 dias), e para F2 as amostras com os tempos iniciais ( 0 a 8 dias) apresentaram diferença significativa entre si. Quanto à perda de ácido ascórbico durante o período de armazenamento, observaram-se as maiores perdas no $8^{\circ}$ dia para F1 $(10,39 \%)$ e no $12^{\circ}$ dia para F2 $(6,11 \%)$, sendo a maior perda total observada para F1 $(33,59 \%)$.

De acordo com Damodaran; Parkin (2017) este elevado valor de ácido ascórbico pode contribuir favoravelmente para sua estabilidade, uma vez que quando presente em altas concentrações, a taxa de degradação desta vitamina é reduzida. Observou-se que para as duas formulações estudadas a concentração de ácido ascórbico tende a ser constante a partir do $24^{\circ}$ dia de armazenamento. Maeda et al. (2007), estudando a estabilidade do ácido ascórbico em néctar de camucamu, observaram que há perdas de $20 \%$ quando armazenado à temperatura ambiente ao final de 150 dias de armazenamento e de 12 a 14\% quando armazenado sob refrigeração, pelo mesmo período. Os mesmos 
autores observaram ainda que a temperatura apresentou forte influência sobre o teor de ácido ascórbico, no entanto, a presença ou ausência de luz não afetou significativamente o teor de ácido ascórbico do néctar de camucamu, comportamento este não avaliado no presente estudo.

Oliveira et al. (2014), ao analisarem a conservação pós-colheita de camu-camu armazenado sob diferentes condições, observaram que o teor de ácido ascórbico não foi influenciado significativamente pelos tratamentos, tendo isso ocorrido apenas pelos dias de armazenamento em que se observou uma queda gradativa ao longo dos 25 dias, havendo uma perda de $20 \%$ desta vitamina. Perdas semelhantes de ácido ascórbico foram na polpa de camu-camu foram descritas por Justi et al. (2000). Oliveira et al. (2013) observaram uma variação no teor de ácido ascórbico em torno de $7 \%$, ao analisarem a polpa de camu-camu submetida à radiação gama.

Yamashita et al. (2003), avaliando a estabilidade de ácido ascórbico em frutos e produtos de acerola, observaram perdas de até $43 \%$ em frutos armazenados a $-12{ }^{\circ} \mathrm{C}$, e de $19 \%$ nos armazenados a $-18{ }^{\circ} \mathrm{C}$ durante 120 dias de estocagem. Perdas significativas de ácido ascórbico também são observadas em produtos desidratados, como observado por Taxi (2001), avaliando o suco de camucamu micro encapsulado, porém nada foi descrito na literatura referente à perda de ácido ascórbico em produtos lácteos armazenados sob refrigeração.

\section{CONCLUSÕES}

Ao analisar as formulações de iogurte do leite de búfala com polpa de camu-camu em duas concentrações distintas foi possível observar que o armazenamento por 28 dias tem efeito expressivo quanto à redução do ácido ascórbico. A formulação F2 apresentou menor perda de ácido ascórbico durante o período de armazenamento mostrando-se como uma boa fonte desse composto no iogurte desenvolvido. No entanto, é imprescindível

Tabela 3 - Teores de ácido ascórbico $(\mathrm{mg} / 100 \mathrm{~g})$ obtido na formulação $\mathrm{F} 1$ com 8,3\% de polpa e formulação F2 com 12,5\% de polpa de camu-camu durante o período de armazenamento sob refrigeração por 28 dias

\begin{tabular}{ccccc}
\hline \multirow{2}{*}{$\begin{array}{c}\text { Tempo de } \\
\text { armazenamento (dias) }\end{array}$} & F1 & Perda (\%) & F2 & Perda (\%) \\
\cline { 2 - 5 } $\mathbf{0}$ & $242,2 \pm 3,81^{\mathrm{a}}$ & - & $317,7 \pm 3,93^{\mathrm{a}}$ & - \\
$\mathbf{4}$ & $235,4 \pm 3,93^{\mathrm{a}}$ & 2,78 & $302,2 \pm 3,81^{\mathrm{b}}$ & 4,89 \\
$\mathbf{8}$ & $211,0 \pm 3,81^{\mathrm{b}}$ & 10,39 & $288,8 \pm 2,91^{\mathrm{c}}$ & 4,43 \\
$\mathbf{1 2}$ & $197,7 \pm 1,53^{\mathrm{c}}$ & 6,32 & $271,3 \pm 3,93^{\mathrm{d}}$ & 6,11 \\
$\mathbf{1 6}$ & $189,8 \pm 1,89^{\mathrm{d}}$ & 3,96 & $264,4 \pm 3,21^{\mathrm{de}}$ & 2,52 \\
$\mathbf{2 0}$ & $182,2 \pm 2,00^{\mathrm{e}}$ & 4,13 & $257,7 \pm 1,26^{\mathrm{e}}$ & 2,52 \\
$\mathbf{2 4}$ & $171,2 \pm 1,81^{\mathrm{f}}$ & 5,95 & $245,7 \pm 4,04^{\mathrm{f}}$ & 4,66 \\
$\mathbf{2 8}$ & $170,3 \pm 1,88^{\mathrm{f}}$ & 0,53 & $242,2 \pm 2,52^{\mathrm{f}}$ & 1,36 \\
\hline Perda total (\%) & - & 33,59 & - & 26,43 \\
\hline
\end{tabular}

* Letras diferentes, na mesma coluna, indicam diferenças significativas pelo teste de Tukey, $(\mathrm{p}<0,05)$. 
a realização de estudos mais aprofundados com o objetivo de identificar quais possíveis variáveis podem estar influenciando nesta perda. Portanto, este produto adicionado de polpa de camu-camu mantém concentrações significativas desta vitamina, podendo ser considerado uma boa fonte da mesma.

\section{REFERÊNCIAS}

BARRETO, A. G. et al. Clarificação de polpa de camu-camu por microfiltração. Brazilian Journal of Food Technology, v. 16, n. 3, p. 207-215, 2013.

BORGES, K. C. et al. Iogurte de leite de búfala sabor Cajá (Spondias lutea L.): Caracterização físico-química e aceitação sensorial, entre indivíduos de 11 a 16 anos. Alimentos e Nutrição, v. 20, n. 2, p. 295$300,2009$.

BRASIL. Ministério da Agricultura, Pecuária e Abastecimento. Instrução Normativa $n^{\circ}$ 46 , de 23 de outubro de 2007. Regulamento técnico de produção, identidade e qualidade de leites fermentados. Diário Oficial da União: seção 1, Brasília, DF, n. 205, p. 4, 24 out. 2007.

COSTA, G. N. S. et al. Desenvolvimento de um iogurte sabor juçaí (Euterpe edulis Mart.): avaliação físico-química e sensorial. Revista Eletrônica TECCEN, v. 5, n. 2, p. 43-58, 2012.

CUNHA NETO, O. C. et al. Avaliação do iogurte natural produzido com leite de búfala contendo diferentes níveis de gordura. Ciência e Tecnologia de Alimentos, v. 3, n. 25 , p. $448-453,2005$.

DAMODARAN, S.; PARKIN, K. L. Química de Alimentos de Fennema. São Paulo: Artmed Editora, 2017. 1085 p.
FUJITA, A. et al. Effects of spray-drying parameters on in vitro functional properties of camu-camu (Myrciaria dubia Mc. Vaugh): a typical Amazonian fruit. Journal of Food Science, v. 82, n. 5, p. 1083-1091, 2017.

HORWITZ, W.; LATIMER Jr., G. W. (ed.). Official methods of analysis of AOAC International. 18th ed. 1st rev. Gaithersburg: AOAC International, 2006.

JUSTI, K. C. et al. Nutritional composition and vitamin $\mathrm{C}$ stability in stored camucamu (Myrciaria dubia) pulp. Archivos Latinoamericanos de Nutricion, v. 50, n. 4, p. 405-408, 2000.

LEE, D. H.; FRANCIS, F. J. Standardization of pigment analyses in cranberries. Hort Science, v. 7, n. 1, p. 83-84, 1972.

LIMA, C. A. Caracterização, propagação e melhoramento genético de pitaya comercial e nativa do Cerrado. Orientador: Fábio Gelape Faleiro. 2013. 124 f. Tese (Doutorado em Agronomia) - Universidade de Brasília, Brasília, 2013.

MAEDA, R. N. et al. Determinação e formulação de néctar de camu-camu (Myrciaria dubia Mc Vaughan). Revista Ciência e Tecnologia de Alimentos, v. 26, n. 1, p. 70-74, 2006.

MAEDA, R. N. et al. Estabilidade de ácido ascórbico e antocianinas em néctar de camucamu (Myrciaria dubia Mc vaugh). Revista Ciência e Tecnologia de Alimentos, v. 27, n. 2, p. 313-316, 2007.

MARINHO, R. R. et al. Caracterização físico-química e microbiológica de xaropes de cupuaçu e camu-camu.In:JORNADA DE INICIAÇÃO CIENTÍFICA PIBICCNPQ/ FAPEAM/ INPA, 18.; 2009, Manaus. Anais [...].Manaus: Instituto Nacional de Pesquisas da Amazônia, 2009. p. 795-799. 
MARQUES, M. P. Processamento, caracterização físico-química e aceitabilidade de xaropes obtidos de frutos de cubiu (Solanun sessiliflorum dunal) e camu camu (Myrciaria dubia McVaugh). 2006. Dissertação (Mestrado em Ciência de Alimentos) - Universidade Federal do Amazonas, Manaus, 2006.

MESQUITA, A. J. et al. Qualidade físicoquímica e microbiológica do leite cru bubalino. Goiânia: UFG/CEGRAF, 2001. 77p.

MODESTO JÚNIOR, E. N. et al. Elaboração de iogurte grego de leite de búfala e influência da adição de calda de ginja (Eugenia uniflora L.) no teor de ácido ascórbico e antocianinas do produto. Revista do Instituto de Laticínios Cândido Tostes, v. 71, n. 3, p. 131-143, 2016.

NOJAVAN, S. et al. Extraction and quantitative determination of ascorbic acid during different maturity stages of Rosa canina L. fruit. Journal of Food Composition and Analysis, v. 21, p. 300-305, 2008.

OLIVEIRA, J. et al. Atmosfera modificada e refrigeração para conservação pós-colheita de camu-camu. Ciência Rural, v. 44, n. 6, p. 1126-1133, 2014.

OLIVEIRA, J. et al. Polpa de camu-camu (Myrciaria dubia) submetida a radiação gama. Acta Amazonica, v. 62, n. 1, p. 7-12, 2013.

OLIVEIRA, K. A. M. et al. Desenvolvimento de formulação de iogurte de ariticum e estudo da aceitação sensorial. Revista Alimentos e Nutrição, Araraquara, v. 19, n. 3, p. 277-281. 2008.

OLIVEIRA, P. D. et al. Avaliação sensorial de iogurte de açaí (Euterpe oleracea Mart) tipo “sundae". Revista do Instituto de Laticínios Cândido Tostes, v. 66, n. 380, p. 5-10, 2011.
PAIVA, Y. F. et al. Iogurte adicionado de polpa de abacaxi, base mel: Elaboração, perfil microbiológico e físico-químico. Revista Verde, v. 10, n. 5, p. 22-26, 2015.

PIMENTEL, C. V. M. B.; FRANCKI, V. M.; GOLLÜCKE, A. P. B. Alimentos funcionais: introdução às principais substâncias bioativas em alimentos. 1. ed. São Paulo: Livraria Varela, 2005. 95 p.

PINEDO, R. Estudo da estabilização da polpa de Camu-camu (Myrciaria dubia (H.B.K.) Mc Vaugh) congelada visando à manutenção de ácido ascórbico e de antocianinas. 2007. Tese (Doutorado em Engenharia Química) - Universidade Estadual de Campinas, São Paulo, 2007.

RIBEIRO, S. I.; MOTA, M. G. C.; CORRÊA, M. L. P. Recomendações para o cultivo do camucamuzeiro no Estado do Pará. Circular Técnica Embrapa, Belém, Pará, 2002.

ROCHA, C. et al. Iogurte de leite de búfala sabor frutos do Cerrado. Boletim do Centro de Pesquisa de Processamento de Alimentos, v. 22, n. 1, p. 97-106, 2004.

RODRIGUES, R. B.; MARX, F. Camu camu [Myrciaria dubia (H. B. K.) Mc Vaugh]: a promising fruit from the Amazon Basin. Nutrition, v. 30, n. 9, p. 376-381, 2006.

ROSA, R. M. S. S. Iogurte de leite de búfala adicionado de polpa de frutas da Amazônia: Parâmetros de qualidade. 2011. $85 \mathrm{f}$. Tese (Doutorado em Higiene Veterinária e Processamento Tecnológico de Produtos de Origem Animal) - Universidade Federal Fluminense, Niterói, RJ, 2011.

SANTOS, A. K. S. Produção de pó de pitaia vermelha (Hylocereus costaricensis) via atomização spray drying para enriquecimento de iorgute tipo grego. 2015. 
113 f. Dissertação (Mestrado em Tecnologia de Alimentos) - Instituto Federal de Educação, Ciência e Tecnologia, Ceará, 2015.

SOARES, C. A. et al. Elaboração e caracterização de iogurte tipo sundae, sabor bacuri (Platoniainsignis Mart.). In: CONGRESSO BRASILEIRO DE CIÊNCIA E TECNOLOGIA DE ALIMENTOS, 25; INTERNATIONAL TECHNICAL SYMPOSIUM, 4.; 2016, Gramado. Anais [...].Gramado: Sociedade Brasileira de Ciência e Tecnologia de Alimentos, 2016.

SOUZA, A. L. R. et al. Concentration of camucamu juice by the coupling of reverse osmosis and osmotic evaporation processes. Journal of Food Engineering, n. 119, p. 7-12, 2013.
TAXI, A. M. C. D. Suco de camu-camu (Myrciaria dubia) microencapsulado obtido através de sacagem por atomização. Tese (Doutorado em Ciência de Alimentos) - Universidade Estadual de Campinas, Campinas, 2001.

YAMASHITA, F. et al. Produtos de acerola: Estudos da estabilidade de vitamina C. Ciência e Tecnologia de Alimentos, v. 23, n. 1, p. 92-94, 2003.

YUYAMA, K.; AGUIAR, J. P. L.; YUYAMA, L. K. O. Camu-camu um fruto fantástico como fonte de vitamina C. Acta Amazônica, v. 32, n. 1, p. 169-174, 2002. 